\title{
Picture Perception: Effects of Luminance on Available Information and Information-Extraction Rate
}

\author{
Geoffrey R. Loftus \\ University of Washington
}

\begin{abstract}
SUMMARY
In each of four experiments, complex visual stimuli-pictures and digit arrays-were remembered better when shown at high luminance than when shown at low luminance. Why does this occur? Two possibilities were considered: first that lowering luminance reduces the amount of available information in the stimulus, and second that lowering luminance reduces the rate at which the information is extracted from the stimulus. Evidence was found for both possibilities. When stimuli were presented at durations short enough to permit only a single eye fixation, luminance affected only the rate at which information is extracted: decreasing luminance by a factor of 100 caused information to be extracted more slowly by a factor that ranged, over experiments, from 1.4 to 2.0 . When pictures were presented at durations long enough to permit multiple fixations, however, luminance affected the total amount of extractable information. In a fifth experiment, converging evidence was sought for the proposition that within the first eye fixation on a picture, luminance affects the rate of information extraction. If this proposition is correct and, in addition, the first eye fixation lasts until some criterion amount of information is extracted, then fixation duration should increase with decreasing luminance. This prediction was confirmed.
\end{abstract}

Luminance is a powerful variable in everyday visual experience-it seems more difficult to perform visual tasks such as searching or reading, under conditions of dim illumination than under conditions of bright illumination. An experiment reported by Loftus (1982) provided some confirmation of this intuition. He presented a series of complex, naturalistic pictures in the study phase of a yes-no recognition procedure. Each picture was presented for either 250 or $1,500 \mathrm{~ms}$, at a luminance that was varied over a $2 \log$ unit range, and was followed by darkness. Subsequent recognition memory was a positive function of both luminance level and of exposure duration.

The salutory effect of increased exposure duration on picture memory has been demonstrated many times (e.g., Loftus, 1972; Loftus \& Kallman, 1979; Loftus, Nelson, \& Kallman, 1983; Potter \& Levy, 1969; Shaffer \& Shiffrin, 1972) and is easy to account for. Any visual stimulus may be conceptualized as containing information. An observer, viewing the stimulus, extracts information from it and builds up a representation of it in memory. The more viewing time, the more information is extracted, and the more complete is the memory representation. The more complete the memory representation, the better is performance on any subsequent memory test.

The effect of luminance on memory for visual material is not as straightforward to explain and has received much less attention than the effect of exposure duration. The present research was carried out for two purposes: first to provide parametric data on the effects of luminance on memory for visual material, and second to identify why these effects occur. Five experiments are reported. In the first four, visual stimulicomplex, naturalistic pictures in Experiments 1-3, and digit arrays in Experiment 4-were presented for varying exposure durations and varying luminances. All stimuli were followed by a mask in order to control the time available for perceptual processing (cf. Sperling, 1963), and subsequently, memory for the stimuli was tested. In the fifth experiment, the effect of luminance on eye fixation durations was investigated. 


\section{How Might Luminance Affect Memory?}

Since target pictures were not masked in the Loftus (1982) experiment, the luminance effect could not have been mediated by target/mask energy. Rather, luminance must have affected extraction of information from the picture itself. There are two broad explanations for how this effect may have occurred. First, decreasing stimulus luminance may have caused an inability to extract some portion of the information upon which the memory test was based. Second, decreasing stimulus luminance, although not affecting the total potentially extractable information, may have caused a decrease in the rate at which the information was extracted.

Each of these explanations implies a specific outcome of any visual memory experiment in which exposure duration ${ }^{1}$ and luminance are factorially combined at the time the stimuli are originally viewed. Suppose first that lowering luminance causes a decrease in the amount of extractable information. In this case, low-luminance memory performance must remain lower than high-luminance memory performance, even at indefinitely long exposure durations. More specifically, consider a curve relating memory performance to exposure duration (hereinafter called a performance curve). A performance curve reflects extraction of information over viewing time. If lowering luminances causes a decrease in extractable information, then performance curves corresponding to different luminance levels must asymptote at different levels.

Suppose, alternatively, that the same information is acquired from stimuli of different luminances, but at a slower rate from lower luminance stimuli. In this case it must require

This research was supported by National Science Foundation Grant BNS82-09617 to the author. I thank Greg Ihli, Carrie Johnson, Bonnie MacDonald, and Mark Reinitz for running the experiments. The implications of plotting performance curves on a log time axis were pointed out to me by Brian Wandell, to whom I am highly indebted. Steve Buck, Earl Hunt, Ellen Markman, Walter Nelson, George Sperling, and an anonymous revicwer provided valuable comments on an earlier version of the manuscript.

Requests for reprints should be sent to Geoffrey Loftus, Department of Psychology, University of Washington, Seattle, Washington 98195. more time to extract a given amount of information from low- relative to high-luminance pictures. More specifically, this explanation predicts that,

$$
P H(t)=P L(k t),
$$

where $P H(x)$ and $P L(x)$ are performance for high- and low-luminance stimuli, respectively, following an exposure duration of $x$, and $k>$ 1. Equation 1 is derived in Appendix 1. Its meaning is that information is extracted slower by a factor of $k$ from low-luminance pictures relative to high-luminance pictures. Therefore, if an exposure duration of $t$ is required for a high-luminance stimulus to achieve the performance level that results from some given amount of information, then a duration of $k t$ will be required for a low-luminance stimulus if the same amount of information is to be acquired and the same performance level achieved.

\section{The Extraction-Rate Hypothesis}

For ease of discourse, the prediction given by Equation 1 -that luminance exerts its influence only on the information-extraction rate-will be termed the extraction-rate hypothesis. This hypothesis can be viewed as a generalization of Bloch's law. Bloch's law states that within some critical period (usually $100 \mathrm{~ms}$ or less), visual behavior depends only on the product of stimulus intensity and stimulus duration-in effect, on the total number of photons from the stimulus that arrive at the eye within the critical period. Bloch's law results from temporal integration of photons-of physical information-early in the visual system. The extraction-rate hypothesis, while encompassing Bloch's law, can also refer to integration of information at a more central level, where such integration is not determined by the rate of incoming physical information. Bloch's law is the special case of Equation 1 in which $k$ equals the ratio of the physical stimulus intensities in the high- and low-luminance conditions.

\footnotetext{
'The predictions will actually apply to processing time, which is not always the same as exposure duration. In the present Experiments 1-4, a mask always followed a target stimulus in order to control processing time as best as possible. The implications of using a mask are discussed later in this article.
} 
Two experiments reported by Turvey (1973, Experiments I and II) used a simplified version of the paradigm used in Experiments 1-4 of the present research. In Turvey's experiments, single letters were presented for brief periods of time, followed by a noise mask, and the minimum target-mask interstimulus interval (ISI) necessary for the target to be reported was measured. As in the present experiments, both target duration and target luminance were varied. Turvey found that performance-minimum ISIdepended only on the product of stimulus duration and stimulus luminance, thereby confirming the special, Bloch's law, version of the extraction-rate hypothesis.

\section{Experiment 1: Old-New Picture Recognition}

In Experiment 1, an old-new recognition procedure was used. In an initial study phase, target pictures were presented, one by one, for inspection. Immediately following the study phase was a test phase in which the target pictures, randomly intermingled with distractor pictures, were presented, again one by one, in an old-new recognition test. Two independent variables, exposure duration and stimulus luminance were factorially combined in the study phase. Exposure duration varied from 50 to $800 \mathrm{~ms}$, and luminance varied over 2 log units. All pictures were followed, immediately at offset, by a noise mask whose purpose was to control the available processing time by eliminating the icon that would otherwise follow a picture.

\section{Method}

Subjects. A hundred and twenty University of Washington undergraduates participated for course credit. They were run in 24 groups of 5 subjects per group.

Stimuli. The targets were 144 naturalistic color pictures, prepared as $35-\mathrm{mm}$ slides, depicting seascapes, landscapes, and cityscapes. They were randomly placed into two slide trays of 72 slides per tray.

The mask consisted of a jumble of straight and curved, black and grey lines on a white background. The mask was much brighter than the targets: When the mask was superimposed over a target picture that was displayed at maximum luminance, the target could not be seen at all. This target-mask luminance configuration, which was used in Experiments 1-4, satisfies the "minimum control" suggested by Eriksen (1980) for experiments in which a mask is used to control perceptual processing time.
Table 1

Stimulus Luminances Used in Experiments I-3 and 5

\begin{tabular}{lc}
\hline \multicolumn{1}{c}{ Stimulus } & Luminance $\left(\mathrm{cd} / \mathrm{m}^{2}\right)$ \\
\hline Adapting field & 0.22 \\
Projector on, no slide & 131.82 \\
Fixation spot & 1.31 \\
Mask & 86.41 \\
Background & 8.82 \\
Black markings & 9.91 \\
Grey markings & \\
\hline
\end{tabular}

All relevant stimulus luminances used in Experiments 1-3 and 5 are shown in Table 1. This table (plus Table 3 for Experiment 4) allows some calculations of stimulus contrast, which covaried with luminance in all five experiments. Since pictures are spatially heterogeneous with respect to luminance, it is not possible to compute a single measure of contrast for Experiments 1-3 and 5, all of which used pictures as stimuli. In Experiment 4, however, alphanumeric stimuli were used and calculation of contrast is thus possible.

Apparatus. The slides were displayed via Kodak random-access carousel projectors and subtended a visual angle that ranged from $15^{\circ}$ to $22^{\circ}$ horizontal and from $10^{\circ}$ to $15^{\circ}$ vertical, depending on where the subject sat. Timing was controlled by Gerbrands tachistoscopic shutters with rise and fall times of approximately $1 \mathrm{~ms}$. Two standard carousel projector were used to present the mask that followed each target and a dim fixation point that preceded each target.

Design. Targets were displayed for one of six exposure durations: $50,100,200,300,400$, and $800 \mathrm{~ms}$. Two values of target luminance were factorially combined with exposure duration. The bright targets were shown at the projector's normal level, that is at $0 \mathrm{log}$ units attenuation (LUA). The dim targets were attenuated by $2.0 \mathrm{log}$ units with a neutral-density filter. There were thus 6 exposure durations $\times 2$ luminance levels for a total of 12 , within-subjects, experimental conditions.

Procedure. An experimental session consisted of a study phase followed by a test phase using the pictures in the first slide tray and then another study and test phase using the pictures in the second slide tray. For each tray, 36 pictures were presented at the time of study. The 12 display conditions occurred in random order with the restriction that each condition occurred once during each of the three, 12-trial blocks within each slide tray.

The sequence of events on each study trial was as follows. First, a 300 -ms tone signaled the subjects to fixate a dim spot which concurrently appeared at the center of the viewing field. A target picture was then presented followed by the mask, which lasted $300 \mathrm{~ms}$. An intertrial interval of $8 \mathrm{~s}$ was followed by the warning tone signaling the start of the next trial.

At the time of test, all 72 pictures in the slide tray were shown in a random order for $6 \mathrm{~s}$ apiece. Test picture luminance was attenuated by $1.0 \mathrm{log}$ units. The test order was different for the two slide trays but for each tray the order was identical over all $\mathbf{2 4}$ groups in the experiment. For each test picture, the subject was asked to respond 
old or new corresponding to whether or not he or she judged the picture to have occurred during the study phase.

Each of the 144 pictures appeared as a target for 12 of the $\mathbf{2 4}$ groups and as a distractor for the remaining 12 groups. Each picture occurred once in each of the 12 study conditions over the 12 groups for which it appeared as a target.

\section{Results and Discussion}

Since the experimental conditions were randomly intermingled during the study phase, there was only a single false-alarm rate, which was 0.26 . Figure 1 , left panel, shows recognition memory $\left(d^{\prime}\right)$ as a function of exposure duration, with separate curves for each of the two luminance levels. The $d^{\prime}$ scores were computed for each condition, for each group, and then averaged over groups.

The major goal in the experiments was to compare shapes of different curves. Standard analysis of variance statistical analyses are therefore inappropriate. Instead, a standard error is presented with each curve. This standard error is computed by dividing the Subject $\times$ Condition interaction variance by the number of subjects, and taking the square root, as described by Loftus and Loftus (1982, pp. 396-400).

As expected from past results (Loftus \& Kallman, 1979; Potter \& Levy, 1969) perfor- mance increased with increasing exposure duration. It is also evident that luminance had a substantial effect on picture recognition: High-luminance pictures were recognized better than low-luminance pictures at all six exposure durations.

The performance benefit enjoyed by highluminance pictures indicates that more information was originally extracted from highluminance pictures relative to low-luminance pictures at all exposure durations. Why does this occur? As noted earlier, two possibilities are considered. First, there might be more extractable information in high-luminance relative to low-luminance pictures. Second, information may be extracted at a faster rate from high-luminance relative to low-luminance pictures.

If more extractable information is available in high-luminance pictures then long-duration, asymptotic performance should be higher for high-luminance relative to lowluminance pictures. It cannot be unambiguously determined from the data in Figure 1 whether asymptotic performance has been reached by $800 \mathrm{~ms}$. The curves certainly appear close to asymptote. The difference between the high-luminance and low-luminance curves did not diminish over exposure durations. A tentative conclusion is that high-

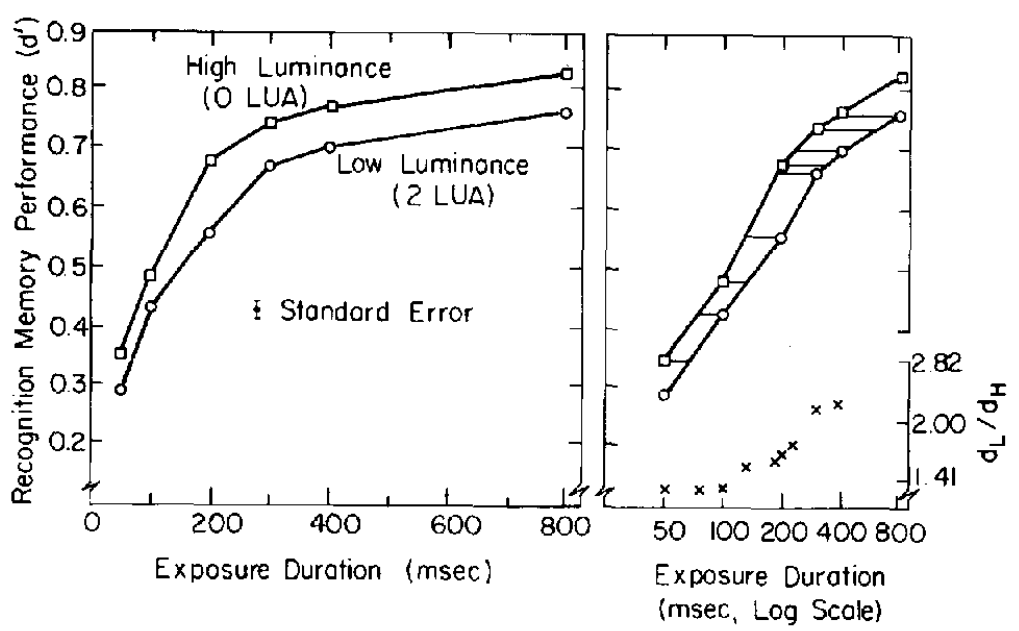

Figure 1. Experiment 1: Recognition memory performance $\left(d^{\prime}\right)$ as a function of exposure duration for high- and low-luminance pictures. (Performance is plotted on a linear time scale in the left panel and on a log time scale in the right panel. The right ordinate in the right panel represents the ratio of low- to high-luminance exposure durations necessary to achieve a particular level of performance. This ratio, as a function of high-luminance duration, is shown by the $x \mathrm{~s}$ at the bottom of the right panel. Each data point is based on 720 observations.) 
luminance pictures contain more extractable information than do low-luminance pictures.

The extraction-rate hypothesis, given by Equation 1, may be easily assessed by plotting performance as a function of $\log$ exposure duration rather than linear exposure duration. The prediction of Equation 1 then becomes

$$
P H[\ln (t)]=P L[\ln (t)+\ln (k)] .
$$

The performance curves corresponding to different luminances should thus be horizontally parallel on a log duration axis. That is, the horizontal difference between the curves should be equal to $\ln (k)$ at all performance levels.

The right panel of Figure 1 shows the data from the left panel, replotted as a function of $\log$ duration. The $x \mathrm{~s}$ at the bottom show the horizontal difference between the two curves, expressed as the ratio of low-luminance to high-luminance exposure duration necessary to achieve a particular level of performance. The results are provocative: This ratio is constant up to a high-luminance duration of $100-200 \mathrm{~ms}$ and then begins to increase. The extraction-rate hypothesis is confirmed for short but not for long exposure durations. The short-duration data may be explained by assuming that the informationextraction system runs slower by a factor of about 1.4 in the low-luminance relative to the high-luminance condition.

It is clear that stimulus luminance has a strong effect on picture recognition. Moreover, the results suggest two ways in which this influence might be mediated. First, the highand low-luminance curves appear to asymptote at different performance levels, which implies that there is more information to be obtained from high-luminance relative to lowluminance pictures. Second, there is, at least at short exposure durations, support for a hypothesis that attributes the effect of luminance only to variation in the informationextraction rate.

A conclusion that there is a short duration/ long duration shift in the effect of luminance on picture processing would not be surprising. The short durations under consideration allow only a single eye fixation, whereas the longer durations allow multiple fixations. Any variable, including luminance, can affect picture processing by affecting extraction of infor- mation within a fixation, and/or by affecting the pattern of eye fixations around the picture. The extraction-rate hypothesis may suffice to describe within-fixation processing, but not across-fixation processing. If, for example, there were peripheral features in the picture that were bright enough to attract the gaze in a high-luminance condition, but not bright enough to attract the gaze in a low-luminance condition, the effect would be of less acrossfixation extractable information in the lowrelative to the high-luminance condition.

Both the asymptotic difference and the short duration data found in Experiment 1 are suggestive, but not conclusive. Experiments 2-4 are designed to investigate asymptotic and short-duration performance more closely.

\section{Experiment 2: Detail Recall}

Experiment 2 was carried out for several reasons. The first was to determine whether high-luminance pictures continue to show higher performance at a longer exposure durations than the ones used in Experiment 1. The second was to investigate a new performance measure. In Experiment 1, there was a problem concerning the appropriate luminance to use at test. How should test luminance relate to the study luminances? Ultimately, the decision was made to use a test luminance that was the geometric mean of the high and low luminance values at study. But this is an imperfect solution. The test luminance may have been psychologically closer to the low or the high study luminance, and could thus have been differentially beneficial to one of the two luminance conditions. To solve this problem, an immediate detail recall procedure rather than a long-term recognition procedure was used in Experiment 2. This procedure is similar to one used by Intraub (1980, Experiment 4) and Loftus and Ginn (1984). Subjects were asked to recall as many details as possible from a picture, immediately after seeing the picture.

\section{Method}

Subjects. Sixty University of Washington undergraduates participated for course credit. They were run in 12 groups of 5 subjects per group.

Stimuli. Sixty new pictures of cityscapes, landscapes, home interiors, and weddings were prepared as $35-\mathrm{mm}$ 
slides. The primary criterion for inclusion of a given picture was that it contain a variety of identifiable, nameable details. The mask was the same one used in Experiment 1.

Apparatus. The apparatus was the same used in Experiment 1.

Design and procedure. As in Experiment 1, the two principal independent variables were target duration and target luminance. The same two luminance levels used in Experiment 1 were used in Experiment 2. Six exposure durations-50,100,200,400,800, and $1600 \mathrm{~ms}$-were factorially combined with the two luminances to produce 12 experimental conditions. Each of the 60 pictures occurred once in each of these 12 conditions over the 12 groups of subjects.

In an experimental session, the 60 pictures were presented one by one. The 12 experimental conditions occurred randomly over the 60 trials with the restriction that, within each block of 12 trials, each condition occurred once. Each trial consisted of the following sequence of events. First, a 1.5-s warning tone signaled the subjects to look at the fixation point. Next came the target picture, followed by a $500-\mathrm{ms}$ mask. Finally, subjects had $20 \mathrm{~s}$ to write down as many details as they remembered from the target. They were instructed to "write down as many details as you can so that a person looking at your list would be able to reproduce the picture as accurately as possible." Following the $20 \mathrm{~s}$ was the warning tone for the next trial.

Following the experiment proper, subjects scored their own data. They were shown the 60 pictures again one by one, and for each picture they wrote down the number of details that they had originally listed. They were told that "a detail" should correspond to a single object listed from the picture. For example, the response "two people" should count as two details. In practice, there were very few cases in which responses were ambiguous. In such cases, subjects were told to use their own judgment.

\section{Results and Discussion}

Subjects had no difficulty carrying out the response task. Nonexistent details were written down in less than $5 \%$ of the trials. The responses almost invariably consisted of the names of objects (e.g., "a person in the middle" or "a boat in the upper left") rather than mere mention of some physical characteristic.

The main results are shown in the left panel of Figure 2. The findings of Experiment 1 are replicated perfectly. Moreover, the difference between the low- and high-luminance conditions is unchanged out to $1,600 \mathrm{~ms}$. Even at that exposure duration, it is not clear that asymptotic level has been reached. However, it does seem unlikely that the curves would converge with yet more exposure time.

To evaluate the extraction-rate hypothesis, the data are replotted on a log duration axis in the right panel of Figure 2. Again, the low- to high-luminance duration ratio necessary to achieve a particular level of performance is indicated by the $x \mathrm{~s}$ at the bottom of the figure. Again, this ratio remains constant at short exposure durations, and then increases.
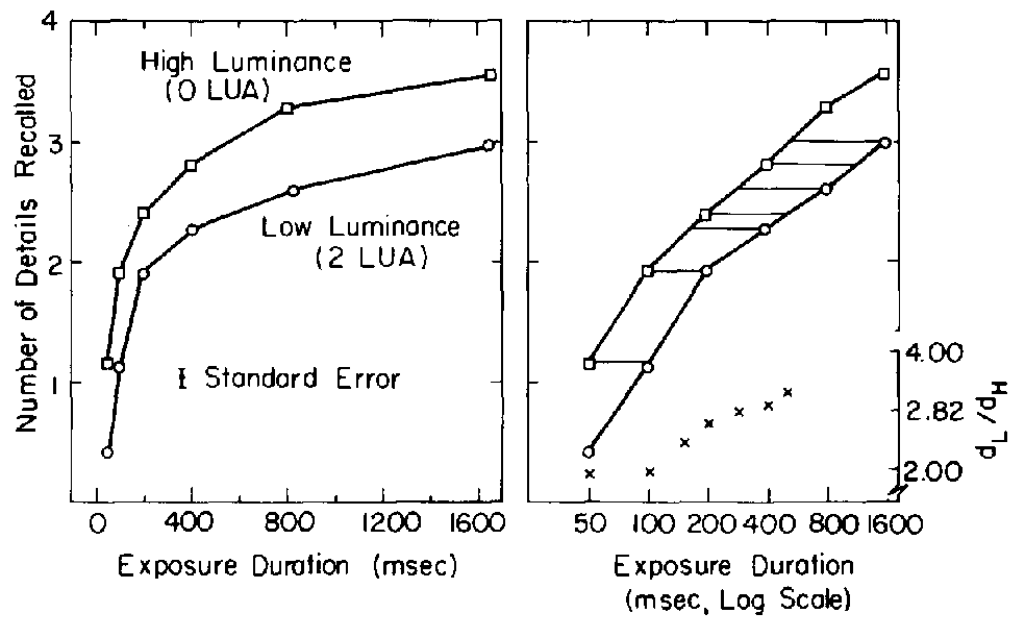

Figure 2. Experiment 2: Number of reported details as a function of exposure duration for high- and lowluminance pictures. (Performance is plotted on a linear time scale in the left panel and on a log time scale in the right panel. The right ordinate in the right panel represents the ratio of low- to high-luminance durations necessary to achieve a particular level of performance. This ratio, as a function of highluminance exposure duration is shown by the $x \mathrm{~s}$ at the bottom of the right panel. Each data point is based on 300 observations.) 
The dependent variable used in Experiment 2-immediate detail recall-measured information extracted from a picture in a way that is free from the problems inherent in an old-new recognition procedure. Despite the use of a new dependent variable, the results of Experiment 1 were precisely replicated. The high- and low-luminance curves appear to asymptote at different performance levels, again suggesting that there is more extractable information in high- relative to low-luminance pictures. At short durations, however, when only a single eye fixation was permitted, the extraction-rate hypothesis was confirmed.

\section{Experiment 3: Detail Recall (Short Exposure Durations)}

Both Experiments 1 and 2 indicate that, at short exposure durations, the ratio of lowluminance to high-luminance duration necessary to achieve a particular level of performance is constant. A possible explanation for this finding is that the extraction-rate hypothesis is appropriate for describing information acquisition within a single eye fixation, but not across eye fixations. However, neither Experiment 1 nor Experiment 2 was expressly designed to investigate performance within a single eye fixation. Experiment 3 is so designed. In Experiment 3, exposure durations were limited to those during which only a single eye fixation would be expected to occur. Also, three rather than two stimulus luminances were used in Experiment 3. As in Experiment 2, a detail-recall test was used to measure the information extracted from the target pictures.

\section{Method}

Subjects. Seventy-five University of Washington undergraduates participated for course credit. They were run in 15 groups of 5 subjects per group.

Stimuli and apparatus. The stimuli and apparatus were the same used in Experiment 2.

Design and procedure. Three levels of stimulus luminance were used: 0,1 , and 2 LUA. There were five stimulus durations within each of the three luminance levels for a total of 15 experimental conditions. One goal in this experiment was to achieve, roughly, the same performance ranges within each of the 3 luminance levels. Therefore, different sets of exposure durations were used within each of the luminance conditions, as shown in Table 2. Each of the 60 slides was shown in each of the 15 conditions over the 15 groups of subjects.
Table 2

Exposure Durations (in Milliseconds) Used in Experiment 3

\begin{tabular}{ccc}
\hline \multicolumn{3}{c}{ Luminance } \\
\hline 0 LUA & $\begin{array}{c}1.0 \text { LUA } \\
\text { (Bright) }\end{array}$ & $\begin{array}{c}2.0 \text { LUA } \\
\text { (Dim) }\end{array}$ \\
\hline 40 & 50 & 65 \\
65 & 95 & 115 \\
90 & 115 & 165 \\
115 & 155 & 215 \\
140 & 190 & 265 \\
\hline
\end{tabular}

Note. $\mathrm{LUA}=\log$ units attenuation.

The experimental procedure was identical to that used in Experiment 2. Within each block of 15 trials, each of the 15 conditions occurred once.

\section{Results and Discussion}

The mean number of recalled details, plotted as a function of $\log$ exposure duration, is shown in Figure 3. There are two noteworthy aspects of these data. First, there is no difference between the 0 and 1 LUA conditions. Second, the horizontal difference between the high-medium luminance curves and the lowluminance curve is invariant over all performance levels.

Experiment 3 demonstrates that, at least at short durations, a change in picture luminance per se is not sufficient to affect the extraction of information from the picture. The two higher luminances of Experiment 3 differed by $1 \log$ unit, yet they produced no difference whatsoever in subsequent memory performance. Evidently, there is a threshold luminance, above which information extraction is not affected by changes in luminance.

The results of Experiment 3 provide support for the assertion that, over stimulus durations from 40 to $265 \mathrm{~ms}$, the extractionrate hypothesis is sufficient to describe the effects of luminance on picture memory. Recall that this hypothesis assumes no difference in the total amount of information extractable from high- versus low-luminance pictures. It attributes performance differences only to differences in the information-extraction rate. 


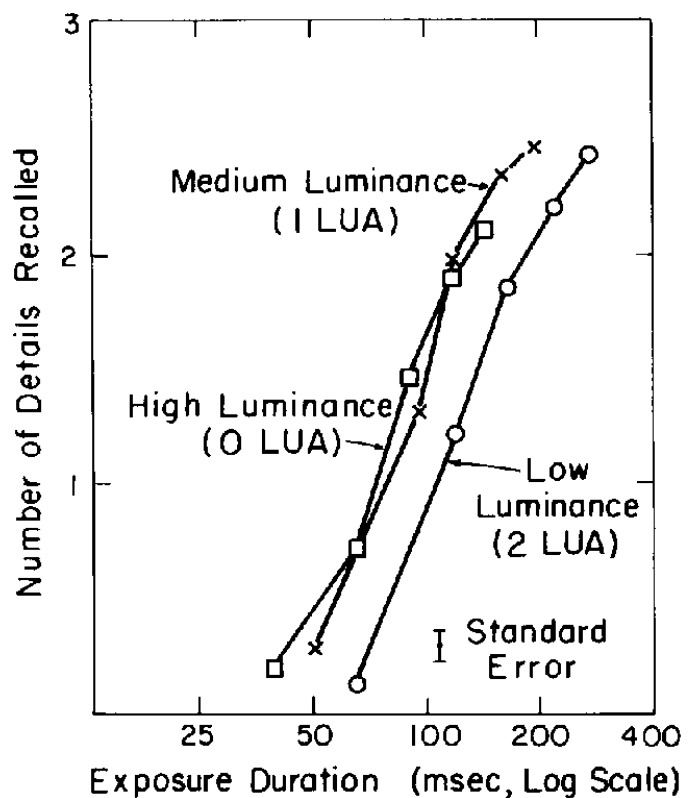

Figure 3. Experiment 3: Number of reported details as a function of exposure duration ( $\log$ scale) for high-, medium-, and low-luminance pictures. (Each data point is based on 300 observations.)

\section{General Discussion: Experiments 1-3}

\section{Overall Luminance Effects}

The results of Experiments 1-3 indicate that luminance sometimes, but not always, affects the amount of information extracted from pictures. In all three experiments, reducing luminance by $2 \log$ units relative to the unattenuated level of the slide projector produced substantial decrements in memory performance (and presumably in extracted information). However, in Experiment 3, an intermediate value of 1 LUA produced no performance deficit.

Adelson and Jonides (1980) varied luminance in a Sperling (1960) delay-of-cue, partial report paradigm in which letters were used as stimuli. They found no effect on either the delay functions or on long-delay asymptotic performance of decreasing luminance from 222.8 to $55.7 \mathrm{~cd} / \mathrm{m}^{2}$, and only a small effect of decreasing luminance further to $27.7 \mathrm{~cd} / \mathrm{m}^{2}$. In a similar paradigm, Keele and Chase (1967) found no effects on memory span (full-report performance) of lowering luminance from 222.8 to $11.78 \mathrm{~cd} / \mathrm{m}^{2}$.
Asymptotic, or full-report performance level, in a Sperling paradigm is comparable with detail recall in the present experiments. Experiment 3 provides a replication of the Adelson and Jonides (1980) and Keele and Chase (1967) results in that no differences were found over a 1 LUA range. However, further reductions in luminance did cause a performance decrement.

There are at least two reasons to expect the effect of luminance on unmasked, alphanumeric stimuli to be somewhat different from its effect on the masked pictures used in the present experiments. First, as luminance is decreased, any contour must, at some point, become unavailable to the visual system. Because of the homogeneous nature of alphanumeric stimuli (e.g., uniform dark letters on a uniform background), the luminance threshold below which all information in the stimulus becomes unavailable must be fairly sharp. The information in pictures, in contrast, is spatially heterogeneous with respect to luminance, and would likely become unavailable over a wider range of luminance.

Second, both Adelson and Jonides (1980) and Keele and Chase (1967) presented their stimuli, unmasked, for $50 \mathrm{~ms}$ which, although a short duration for a picture, is a long duration for an array of alphanumeric characters (cf. Sperling, 1960, 1963; Sperling, Budiansky, Spivak, \& Johnson, 1971). It is likely that all the information that could become available would become available during that time, even with a slower information-extraction rate that might accrue from a lower luminance level. Indeed, asymptotic/full-report performance in both experiments was about 4-5 items, which is generally the maximum number of items that can ever be reported following a brief presentation.

\section{How Luminance Exerts its Effect}

The present results provide evidence for two separate ways in which the effects of luminance on picture perception are mediated. In Experiments 1 and 2, asymptotic performance differed for the two luminance levels, which suggests that one effect of decreasing luminance is to reduce the amount of information that is extracted across a series of eye fixations on a picture. However, 
the results of all three experiments indicated that at short exposure durations, where only a single eye fixation is possible, the effect of luminance can be attributed to its influence on information-extraction rate only. The suggestion, in other words, is that the same information is acquired within the first eye fixation, independent of luminance, at least over the range of luminances that we have so far examined. The information is just acquired slower if the luminance is sufficiently low.

\section{Experiment 4: Digit Recall}

Experiments 1-3 indicate that the extraction-rate hypothesis is sufficient to describe the effect of luminance during the first eye fixation on a picture. This hypothesis relies on the assumption that the same information is extracted from both high-luminance and low-luminance pictures. This assumption may, however, be incorrect. Perhaps different information was extracted at different rates from pictures of different luminances, but in such a way that the predictions of the hypothesis were confirmed coincidentally. This possibility is difficult to assess when naturalistic pictures are used as stimuli, because the total extractable information in such pictures is not readily identifiable.

To address this issue, digit arrays instead of naturalistic pictures were used as stimuli in Experiment 4. The luminance levels were arranged such that all of the to-be-reported information was available: when the arrays were continuously visible, all digits could be reported perfectly at each of the two luminance levels used in the experiment. Any effect of luminance on performance must therefore be mediated by the speed of extracting information from the stimulus. This allows a more precise test of the extraction-rate hypothesis.

\section{Method}

Subjects. Three subjects, the author and two colleagues, served as subjects. All three subjects were conversant with the experimental issues under investigation, and all had had extensive practice with the task and stimuli used in the experiment.

Stimuli. The stimuli were 72, 12-digit arrays, prepared as black-on-white $35-\mathrm{mm}$ slides. The digits in each array were arranged in three rows of four digits per row. Each digit subtended a visual angle of $0.56^{\circ}$ vertical $\times 0.28^{\circ}$ horizontal. Digits were separated by $0.37^{\circ}$ vertically and $0.74^{\circ}$ horizontally.

The digits in each array were chosen randomly with the restriction that no digit could appear more than twice in any row. The mask used in Experiment 4 was similar to the one used in Experiments 1-3, but contained only black noise on a white background. When the mask was superimposed on the digit arrays projected at the brightest luminance used in the experiment, no digits could be read from the arrays.

An adapting field of $0.97 \mathrm{~cd} / \mathrm{m}^{2}$ was present at all times. Table 3 shows the background and digit luminances in the bright and dim conditions, as well as the mask luminances.

Apparatus. The same projection apparatus used in Experiments 1-3 were used in Experiment 4, except that (a) the apparatus was enclosed in a soundproof box and (b) stimulus display and response collection were controlled by an APPLE II computer system.

Design and procedure. The experiment was performed in 72-trial blocks. On each trial, an array was shown at one of six exposure durations: $50,100,150,200,250$, or $300 \mathrm{~ms}$ and at one of the two luminances. Following each array presentation, the subject reported as many as possible of the digits from one of the three rows. The tobe-reported row was signaled prior to the start of each trial.

More specifically, the following sequence of events occurred on each trial. First, a series of ten, $30 \mathrm{~ms}$ on $/ 30 \mathrm{~ms}$ off, $1000-\mathrm{Hz}$ beeps signaled the beginning of a trial. During this warning period, a fixation point was projected at the point where the middle of the upcoming array would be. There was then a blank (adapting field only) delay of $500 \mathrm{~ms}$, followed by a $200-\mathrm{ms}$ tone that signaled which row in the upcoming array was to be reported: the top, middle, and bottom rows were signaled by tones of 2000,1000 , and $250 \mathrm{~Hz}$, respectively. There was then another 500 -ms blank delay. The array was then presented, followed by the mask, which was displayed for $500 \mathrm{~ms}$. The subject then attempted to type in the four digits from the appropriate row, in order, into a response box, guessing if necessary. Feedback followed each response in the form of four, 250-ms tones: each tone was $1500 \mathrm{~Hz}$ if the corresponding digit had been correctly reported, and $250 \mathrm{~Hz}$ if it had been incorrectly reported. Following feedback was a 500-ms pause prior to the warning signals for the next trial.

Within each block, the 36 conditions formed by a factorial combination of six exposure durations, two luminances, and three rows were presented randomly over the 72 trials, with the restriction that each condition be shown once during the first 36 trials, and once during the second 36 trials. Subjects 1-3 participated in 11,10 , and 7,72 -trial blocks, respectively.

\section{Results and Discussion}

The mean number of correctly reported digits in each of the 12 luminance $X$ exposure duration conditions was computed for each of the 3 subjects, then averaged across subjects. The results are shown in Figure 4, which is arranged similarly to Figures 1-3: 
Table 3

Stimulus Luminances $\left(\mathrm{cd} / \mathrm{m}^{2}\right)$ in Experiment 4

\begin{tabular}{cccccc}
\hline & \multicolumn{2}{c}{ Targets } & & \multicolumn{2}{c}{ Mask } \\
\cline { 2 - 3 } \cline { 5 - 6 } Luminance condition & Background & Digits & & Background & Noise \\
\hline Bright & 51.91 & 13.04 & & 51.91 & 13.04 \\
Dim & 1.72 & 1.08 & & 51.91 & 13.04 \\
\hline
\end{tabular}

performance is plotted as a function of $\log$ exposure duration. Performance on $50-\mathrm{ms}$ low-luminance arrays are at chance (approximately 0.34 digits), whereas performance on 50 -ms high-luminance arrays are slightly above chance. By $300 \mathrm{~ms}$, performance in both luminance conditions is close to perfect. The errors that did occur at these long durations were due in large part to motor errors that subjects made when striking the somewhat sensitive response-box keys.

It is easy to see that the two duration curves are horizontally parallel. Low-luminance arrays required a duration approximately 1.5 times as long as high-luminance arrays to reach any given performance level.

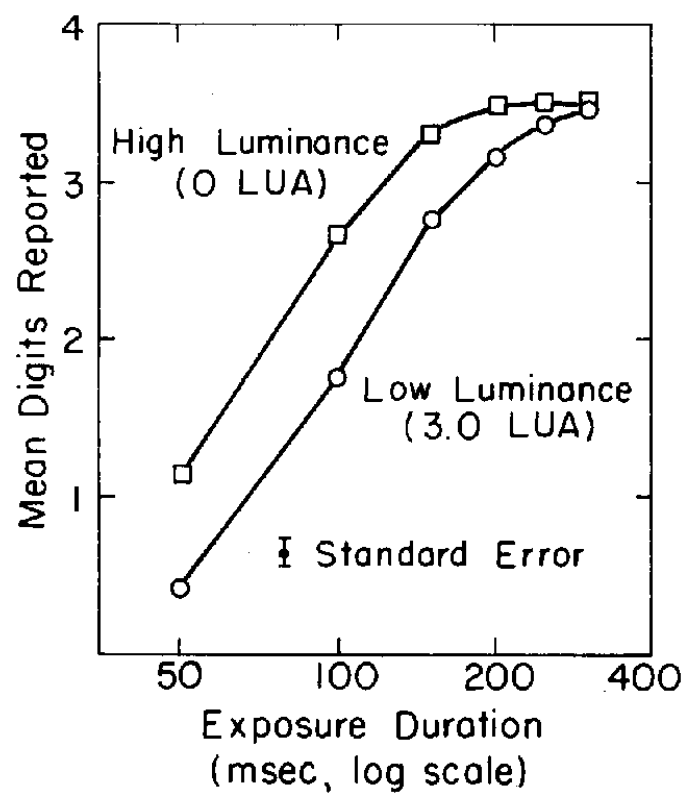

Figure 4. Experiment 4: Number of reported digits as a function of $\log$ exposure duration for high- and lowluminance digit arrays. (Each data point is based on 168 observations.)
Table 4 presents serial position data. It shows the probability of correctly reporting the digit in each of the four positions within the row, for each of the 12, exposure duration $\times$ luminance, experimental conditions. There is evidence that the digits are read in a left-to-right order; the rightmost digit is generally reported with the lowest probability. Of importance is that there are no qualitative differences in serial position effects between dim and bright pictures. Compare, for example, two conditions - the 100-ms bright and $150-\mathrm{ms}$ dim conditions - that show roughly equal overall performance. The serial position data for these two conditions are quite similar.

A substantial effect of stimulus luminance on digit recall was found in Experiment 4. This result contrasts with that of Adelson and Jonides (1980) and Keele and Chase (1967) who found no effect of luminance on

Table 4

Probability of Correct Digit Report as a Function of Serial Position in Experiment 4

\begin{tabular}{ccccc}
\hline \multirow{2}{*}{$\begin{array}{c}\text { Exposure } \\
\text { duration } \\
(\mathrm{ms})\end{array}$} & 1 & 2 & 3 & 4 \\
\cline { 2 - 5 } & \multicolumn{5}{c}{ Serial Position } \\
\multicolumn{5}{c}{ High-luminance arrays } \\
50 & 0.36 & 0.44 & 0.21 & 0.16 \\
100 & 0.82 & 0.76 & 0.61 & 0.51 \\
150 & 0.93 & 0.91 & 0.79 & 0.73 \\
200 & 0.96 & 0.95 & 0.91 & 0.69 \\
250 & 0.98 & 0.96 & 0.86 & 0.70 \\
300 & 0.96 & 0.94 & 0.89 & 0.70 \\
\hline & Low-luminance arrays & \\
50 & 0.09 & 0.09 & 0.13 & 0.12 \\
100 & 0.61 & 0.59 & 0.35 & 0.26 \\
150 & 0.85 & 0.80 & 0.65 & 0.50 \\
200 & 0.91 & 0.87 & 0.78 & 0.65 \\
250 & 0.95 & 0.91 & 0.81 & 0.73 \\
300 & 0.95 & 0.93 & 0.85 & 0.77 \\
\hline
\end{tabular}


asymptotic performance or full report of alphanumeric stimuli. As noted earlier, there are at least two possible reasons for this difference. First, the lowest luminance level used in the present experiments was substantially lower than the lowest luminance used by Adelson and Jonides or Keele and Chase. Second, in these previous experiments, arrays were presented for $50 \mathrm{~ms}$, unmasked, which may well have been enough time to extract all possible extractable information from the array.

The main purpose of Experiment 4 was to test the extraction-rate hypothesis when amount of extractable, task-relevant information in high- and low-luminance stimuli is held constant. Several aspects of the data support the hypothesis. First, the high- and low-luminance performance curves show the predicted constant-ratio, horizontal divergence. Second, a more fine-grained analysis, of serial position effects, indicated qualitative similarity between high- and low-luminance arrays. If exposure durations are chosen such that high- and low-luminance performance is roughly similar, then the serial position effects are also similar. This suggests that, as required by the extraction-rate hypothesis, equal performance in high- and low-luminance conditions is based on extraction of the same information.

\section{Experiment 5: Eye-Fixation Durations}

A new question was asked in Experiment 5: Does picture luminance affect the duration of eye fixations on the picture? A large body of research has been concerned with what causes variation in fixation duration. Although there is no comprehensive answer to this puzzle, there is general agreement that variation is at least partly attributable to the amount of time required to extract information during the fixation (e.g., Just \& Carpenter, 1980; see Loftus, 1983, and Rayner, 1978, for reviews).

The short-duration data of Experiments 1-3 may be accounted for by assuming that, during the first fixation on a picture, luminance affects the rate at which information is extracted. If lower luminances lead to the same information being acquired on the first fixation, but at a slower rate, then the duration of the first fixation should increase with decreasing luminance.

\section{Method}

Subjects. Four University of Washington undergraduates, recruited by a newspaper advertisement, served as subjects. They were paid $\$ 5$ apiece for a session that lasted approximately an hour.

Stimuli. The 144 slides used in Experiment 1, plus 16 additional, similar slides, for a total of 160 , were used in Experiment 5.

Apparatus. The apparatus is described in detail by Loftus (1979). Briefly, pictures were displayed via a Kodak carousel projector with a tachistopic shutter identical to those used in Experiments 1-4. Eye movements were recorded with a corneal reflection device described by Mackworth (1967). This device yields a television picture of the scene being observed by the subject, superimposed over which is a spot of light that moves about the scene in accordance with where the subject is fixating. The eye movement data thus obtained were recorded on videotape.

Design and procedure. Pictures were shown, one by one, for $1.5 \mathrm{~s}$ apiece. Subjects were told to look at the pictures as if they were to receive a subsequent recognition memory test. Of the 160 pictures, 40 were shown in each of four luminance conditions: $0.6,1.6,2.2$, and 2.6 LUA. Luminance was blocked in 20-trial blocks. For each subject, the four luminances were presented in an abcddcba design over the eight 20-trial blocks. Luminance was counterbalanced such that each picture was shown in each of the four luminance conditions over the 4 subjects. The 160 pictures were shown in the same order for the 4 subjects; thus, each luminance level occurred once in each of the 20 -trial blocks over the 4 subjects.

On each trial, the following series of events took place. First, the experimenter said "ready," which signaled the subject to look at a fixation point. When the experimenter (who could monitor fixation location) determined that the subject was fixating the fixation point, he displayed the picture. Following the picture was a 500 -ms mask. After each trial, the subject was asked to refixate the fixation point, and the experimenter carried out any recalibration that was necessary. The subject was allowed to rest whenever he or she wished.

\section{Results and Discussion}

To analyze the data, the videotape was played at approximately $1 / 10$ its normal speed. The experimenter viewed the tape in conjunction with a real-time computer program. The experimenter signaled the program (with a keypress) whenever he detected a saccade on the videotape. Special characters were used to signal the beginning and end of a picture. The program recorded the actual times between the signal imputs and then, based on the original exposure duration of $1.5 \mathrm{~s}$, normalized all times to arrive at the actual fixation durations. 
Figure 5 shows fixation duration as a function of luminance level for the first, second, and third fixations on the picture. For each of the three fixations, duration increased with decreasing stimulus luminance. The effect of luminance was greatest on the first fixation. ${ }^{2}$

The data for the second and third fixations cannot be unambiguously interpreted. Whereas the location of the first fixation was always controlled by the predisplay fixation point, the location of subsequent fixations was uncontrolled and may have systematically depended on luminance level.

This problem notwithstanding, Figure 5 indicates that the effect of luminance on fixation duration becomes progressively smaller with each successive fixation. Why might this occur? One possibility is that a particular feature in a picture is not fixated unless the brightness of that feature is above some criterion. As overall picture luminance is decreased, different, relatively brighter features of the picture are thus fixated. If this were true then, across the four luminance conditions, the luminance variation of fixated features would be less than the experimentally defined $2 \log$ unit variation of the overall picture. This explanation accounts for the decreased effect of luminance on durations of fixations subsequent to the first. However, additional assumptions would be needed to account for the smaller effect of luminance on the duration of the third, relative to the second, fixation.

In any event, the duration of the first fixation increases quite dramatically with decreasing luminance level. This finding is consistent with the hypothesis that luminance does not affect the total amount of information acquired on the first fixation, but only the rate at which the information is acquired.

The hypothesis is not, of course, required by the finding. It is also possible that less information is acquired with lower luminance, but at a sufficiently slower rate that fixation duration still increases with decreasing luminance.

To tie the results of Experiment 5 to those of Experiments 1-3, consider a very simple extension of the information-extraction hypothesis that incorporates the following assumptions:

1. Luminance affects only the rate at which information is extracted.

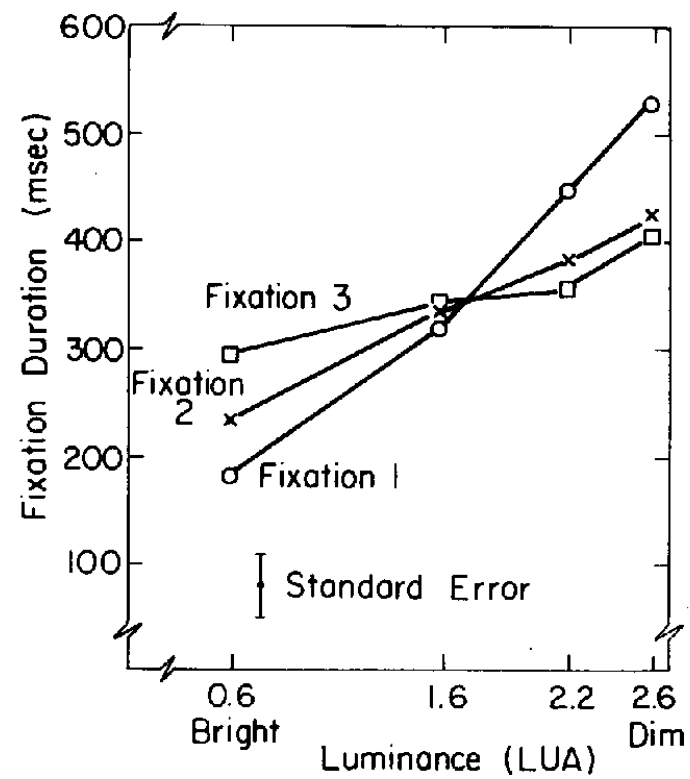

Figure 5. Experiment 5: Eye fixation duration as a function of luminance for the first, second, and third fixation on the picture. (Each data point is based on 160 observations.)

2. The first fixation continues until, and only until, some criterion amount of information is extracted.

3. This criterion is independent of luminance.

Now consider any two luminance levels, high and low. The model just described predicts that on a memory test, the ratio of lowto high-luminance durations necessary to achieve any given level of performance is some constant, $k$, and that the ratio of lowto high-luminance fixation durations is equal to this same $k$.

Experiment 5 was not performed with Experiments 1-3 in mind; hence the experimental conditions were not designed specifically to test this prediction. It is nonetheless possible to see if the prediction is approximately confirmed. Table 5 shows the relevant duration ratios. The ratios stemming from the memory experiments are of the same

\footnotetext{
${ }^{2}$ The duration of the first fixation includes only that part of the fixation that follows the onset of the stimulus. This means that the absolute durations of the first fixation arc not strictly comparable to the durations of subsequent fixations. Of principal interest, however, is the effect of luminance on fixation duration rather than the effect of ordinal fixation number on fixation duration.
} 
Table 5

Low- to High-Luminance Duration Ratios

Necessary to Achieve a Particular Level of

Performance (Experiments 1-3) and Low- to

High-Luminance First Fixation Duration Ratios (Experiment 5)

\begin{tabular}{|c|c|c|c|}
\hline $\begin{array}{l}\text { Experi- } \\
\text { ment }\end{array}$ & & $\begin{array}{l}\text { Low/High } \\
\text { luminance }\end{array}$ & $\begin{array}{c}\text { Duration } \\
\text { ratio }\end{array}$ \\
\hline 1 & 2 & LUA/0 LUA & $1.41: 1$ \\
\hline 2 & 2 & LUA/0 LUA & $2.00: 1$ \\
\hline 3 & 1 & LUA/0 LUA & $1.00: 1$ \\
\hline 3 & 2 & LUA/1 LUA & $1.41: 1$ \\
\hline 5 & 1.6 & LUA/0.6 LUA & $1.68: 1$ \\
\hline 5 & 2.6 & LUA $/ 1.6$ LUA & $1.65: 1$ \\
\hline
\end{tabular}

Note. LUA $=\log$ units attenuation.

magnitudes as the ratios stemming from the fixation duration experiment; they are in the range of 1.4-2.0. This model thus seems worth pursuing.

\section{General Discussion}

\section{The Effect of Luminance}

The most general goal of these experiments was to determine whether memory performance for a visual stimulus is affected by luminance. Experiments 1-4 indicated that when luminance is reduced by 2 log units, relative to the unattenuated level of the slide projector, memory performance is impaired. This effect was observed using both a delayed recognition test (Experiment 1), an immediate detail-recall test (Experiments 2-3), and a digit-recall test (Experiment 4).

There is evidence that luminance exerts its influence in two ways. First, the total amount of extractable information is effectively reduced when luminance is sufficiently low. A likely reason for this is that fewer features in the picture are fixated under conditions of low relative to high luminance.

If exposure duration is restricted such that only a single eye fixation is permitted, then the amount of potentially extractable information is not affected by luminance. Rather, the same information is acquired from lowluminance pictures relative to high-luminance pictures; it is just acquired slower, in accordance with the extraction-rate hypothesis. The results of Experiment 5 indicate that decreasing a picture's luminance causes an increase of the duration of the first eye fixation on the picture. This finding constitutes converging evidence, at least for the general proposition that information acquired on the first fixation is acquired slower with lower luminance levels.

\section{Compensatory Processes}

Confirmation of the extraction-rate hypothesis implies that the cognitive system can compensate for a luminance decrement by operating for a longer time. As noted earlier, the extreme form of this kind of compensatory ability is described by Bloch's law wherein there is an absolute trade off between stimulus duration and stimulus intensity. Turvey (1973, Experiments I and II) has invoked Bloch's law to account for masking by noise of a single letter. He found that the shortest target-mask ISI at which the target was immune to the effect of the mask was determined by the product of stimulus duration and stimulus intensity.

There is a good deal of similarity between Turvey's experiments and the present ones. In both sets of studies, extraction of information from masked targets was at issue. In both sets, different combinations of time and luminance necessary for some criterion performance was measured. And, in both sets, the time-luminance trade-off law described by Equation 1 was confirmed.

There is also, however, a major difference between Turvey's results and the present ones. Turvey found support for an absolute tradeoff between time and luminance: a luminance decrement of some factor required a duration increment by an identical factor for compensation. In the present experiments, however, luminance is, in a sense, a much less powerful variable than duration: a 100:1 decrement in luminance can be compensated for by, at most, a 2:1 increment in duration. This luminance-duration relation is an instance of what Sperling (1979) refers to as "supersummation." Supersummation is defined to be a situation in which a long-duration stimulus requires less total energy than a shortduration stimulus in order to achieve some criterion performance. Sperling notes that "The essence [of a situation that will produce supersummation] is the intrusion of high- 
order mental processes that require time for their execution, and are relatively indifferent to intensity" (p. 279).

In a sense, Sperling's remark is applicable to the difference between Turvey's and the present experimental paradigms. In Turvey's paradigm, the task of the subject was simple: only one letter had to be identified. Allocating all resources to this one task permitted visual information to be utilized by the cognitive system at a very rapid rate-indeed, the lower bound on cognitive processing rate was set by the rate at which information arrived from the environment. In the present experiments, the subject's task was much more complex. In the digit-memory task of Experiment 4 , subjects had to isolate the target row in the digit matrix and extract information corresponding to 4 digits. In the other experiments, the required processing during the first fixation was even more complex. It is evident that during the first fixation, a picture's gist is identified (cf. Biederman, 1972; Intraub, 1981, Potter, 1975) and specific details are sought out and identified. The degree of cognitive processing needed for this complex task creates a bottleneck: the system cannot use information at the rate at which it enters from the environment, and information integration is therefore slower.

\section{The Role of Masking}

Luminance has often been used as a tool to investigate the effects of visual masking. In some masking experiments, target luminance has been varied (Turvey, 1973; Cheatham, 1952; Kinsbourne \& Warrington, $1962 \mathrm{a}, 1962 \mathrm{~b})$ and in others, mask luminance has been varied (Eriksen, 1966; Eriksen \& Lappin, 1964; Loftus \& Ginn, 1984; Spencer \& Shuntich, 1970; Thompson, 1966). Irrespective of whether it is target or mask energy that is varied, performance has been found to increase with increases in target-mask energy ratio.

The results of the present experiments might be interpreted as simply another replication of these past results: as the targetmask energy ratio increased, memory performance for pictures and digit arrays likewise increased. However, such an interpretation would not account for the findings of Loftus
(1982) who found positive effects of stimulus luminance on picture recognition in the absence of masking, nor would it account for the results of the present Experiment 5 in which eye fixation duration was affected by stimulus luminance.

Masking was not of primary concern in the present experiments. Rather, the experiments were performed under the assumption that target luminance exerts an important effect on information extraction from the target stimulus itself. A mask was used not to investigate masking, but as a tool to control the time available to extract perceptual information from the stimulus (Sperling, 1963). Such control is, of course, necessary to allow a test of the extraction-rate hypothesis.

\section{References}

Adelson, E. H., \& Jonides, J. (1980). The psychophysics of visual storage. Journal of Experimental Psychology: Human Perception and Performance, 6, 486-493.

Biederman, I. (1972). Perceiving real-world scenes. Science, $177,77-80$.

Cheatham, P. G. (1952). Visual perceptual latency as a function of stimulus brightness and contour shape. Journal of Experimental Psychology. 43, 369-380.

Eriksen, C. W. (1966). Temporal luminance summation effects in backward and forward masking. Perception \& Psychophysics, 1, 87-92.

Eriksen, C. W. (1980). The use of a visual mask may seriously confound your experiment. Perception \& Psychophysics, 28, 89-92.

Eriksen, C. W., \& Lappin, J. S. (1964). Luminance summation-contrast reduction as a basis for certain forward and backward masking effects. Psychonomic Science, 1, 313-314.

Intraub, H. (1980). Presentation rate and the representation of briefly glimpsed pictures in memory. Journal of Experimental Psychology: Human Learning and Memory, 6, 1-12.

Intraub, H. (1981). Rapid conceptual identification of sequentially presented pictures. Journal of Experimental Psychology: Human Perception and Performance, 7, 604-610.

Just, M. A., \& Carpenter, P. A. (1980). A theory of reading: From eye fixations to comprehension. Psychological Review, 87, 329-354.

Keele, S. W., \& Chase, W. G. (1967). Short-term visual storage. Perception \& Psychophysics, 2, 383-386.

Kinsbourne, M., \& Warrington, E. K. (1962a). The effect of an aftercoming random pattern on the perception of brief visual stimuli. Quarterly Journal of Experimental Psychology, 14, 223-234.

Kinsbourne, M., \& Warrington, E. K. (1962b). Further studies on the masking of brief visual stimuli by a random pattern. Quarterly Journal of Experimental Psychology, 14, 235-245. 
Loftus, G. R. (1972). Eye fixations and recognition memory for pictures. Cognitive Psychology, 3, 525551.

Loftus, G. R. (1979). On-line eye-movement recorders: The good, the bad, and the ugly. Behavior Methods Research and Instrumentation, 11, 188-191.

Loftus, G. R. (1982). Picture memory: Data and methodology. In C. R. Puff (Ed.), Handbook of Research Methods in Human Memory and Cognition. New York: Academic Press.

Loftus, G. R. (1983). Eye fixations on scenes and text. In K. Rayner (Ed.), Eye Movements in reading: Perceptual and language processes. New York: Academic Press.

Loftus, G. R., \& Ginn, M. (1984). Perceptual and conceptual masking of pictures. Iournal of Experimental Psychology: Learning, Memory, and Cognition, 10, 435-441.

Loftus, G. R., \& Kallman, H. J. (1979). Encoding and use of detail information in picture recognition. Journal of Experimental Psychology: Human Learning and Memory, 5, 197-211.

Loftus, G. R., \& Loftus, E. F. (1982). Essence of statistics. Monterey: Brooks-Cole.

Loftus, G. R., Nelson, W. W., \& Kallman, H. J. (1983). Differential acquisition rates for different types of information from pictures. Quarterly Journal of $E_{X}$ perimental Psychology, 35A, 187-198.

Mackworth, N. H. (1967). A stand camera for line-ofsight recording. Perception \& Psychophysics, 2, 119 127.
Potter, M. C. (1975). Meaning in visual search. Science, 187, 965-966.

Potter, M. C., \& Levy, E. I. (1969). Recognition memory for a rapid sequence. of pictures. Journal of Experimental Psychology, 81, 10-15.

Rayner, K. (1978). Eye movements in reading and information processing. Psychological Bulletin, 85, 618660 .

Shaffer, W. O., \& Shiffrin, R. M. (1972). Rehearsal and storage of visual information. Journal of Experimental Psychology, 92, 292-295.

Spencer, T. J., \& Shuntich, R. (1970). Evidence for an interruption theory of backward masking. Journal of Experimental Psychology, 85, 198-203.

Sperling, G. (1960). The information available in brief visual presentations. Psychological Monographs, 74, $1-29$.

Sperling, G. (1963). A model for visual memory tasks. Human Factors, 5, 19-31.

Sperling, G. (1979). Critical duration, supersummation, and the narrow domain of strength-duration experiments. The Behavioral and Brain Sciences, 2, 279.

Sperling, G., Budiansky, J., Spivak, J., \& Johnson, M. C. (1971). Extremely rapid visual search: The maximum rate of scanning letters for the presence of a numeral. Science, 174, 307-311.

Thompson, J. H. (1966). What happens to the stimulus in backward masking? Journal of Experimental Psychology, 7l, 580-586.

Turvey, M. T. (1973). On peripheral and central processes in vision: Analysis of masking with patterned stimuli. Psychological Review, 80, 1-52.

\section{Appendix 1}

To derive Equation 1 in the text, let

and

$$
r_{L}\left(t_{L}\right)=d I_{L} / d t_{L}=f\left(I_{L}\right)
$$

$$
r_{H}\left(t_{H}\right)=d I_{H} / d t_{H}=k f\left(I_{H}\right),
$$

where $r_{I}(t)$ and $r_{H}(t)$ are the rates of information extraction $(d I / d t$, the derivative of information with respect to time), $k$ is a constant, $I$ is extracted information, and $f$ is some function. The subscripts $L$ and $H$ refer to low- and high-luminance conditions, respectively.

From Equations 1 and 2,

$$
g\left(I_{L}\right) d I_{L}=d t_{L}
$$

and

$$
g\left(I_{H}\right) d I_{H}=k d t_{H},
$$

where $g(I)=1 / f(I)$.
Integrating Equations 3 and 4 ,

$$
G\left(I_{L}\right)=t_{L}
$$

and

$$
G\left(I_{H}\right)=k t_{H},
$$

where $G$ is the integral of $g$. Letting $I_{L}=I_{I I}$, $G\left(I_{L}\right)=G\left(I_{H}\right)$ and therefore, $t_{L}=k t_{H}$. That is, when high- and low-luminance information-and thus, high- and low-luminance performance-is the same, low-luminance duration, $t_{L}$, must be $k$ times high-luminance duration, $t_{H}$.

Received August 8, 1984

Revision received January 21, 1985 